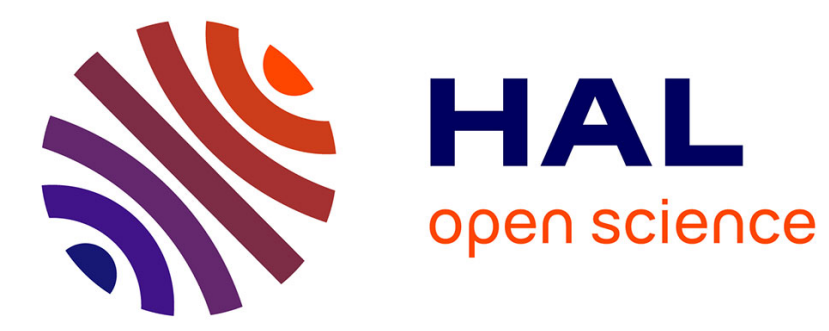

\title{
Static analysis and design strategy of two antagonistically actuated joints
}

Vimalesh Muralidharan, Philippe Wenger, Matthieu Furet

\section{To cite this version:}

Vimalesh Muralidharan, Philippe Wenger, Matthieu Furet. Static analysis and design strategy of two antagonistically actuated joints. New Trends in Mechanism and Machine Science, 2020. hal-03010558

\section{HAL Id: hal-03010558 \\ https://hal.science/hal-03010558}

Submitted on 17 Nov 2020

HAL is a multi-disciplinary open access archive for the deposit and dissemination of scientific research documents, whether they are published or not. The documents may come from teaching and research institutions in France or abroad, or from public or private research centers.
L'archive ouverte pluridisciplinaire HAL, est destinée au dépôt et à la diffusion de documents scientifiques de niveau recherche, publiés ou non, émanant des établissements d'enseignement et de recherche français ou étrangers, des laboratoires publics ou privés. 


\title{
Static analysis and design strategy of two antagonistically actuated joints
}

\author{
Vimalesh Muralidharan ${ }^{1}$, Philippe Wenger ${ }^{1,2}$, and Matthieu Furet ${ }^{1,2}$ \\ 1 Ecole Centrale de Nantes, 44321 Nantes, France \{m.vimalesh94@gmail.com\} \\ 2 Laboratoire des Sciences du Numérique de Nantes (LS2N), CNRS, 44321 Nantes, \\ France $\{$ Philippe.Wenger@ls2n.fr, Matthieu.Furet@ls2n.fr\}
}

\begin{abstract}
This paper compares the static performances of two types of antagonistically actuated joints: a revolute $(\mathrm{R})$ joint and an antiparallelogram (X) joint. Both joints are equipped with lateral springs and actuated with two opposite cables running through the springs. The comparative study is conducted on the basis of their wrench-feasible workspace and stiffness. A methodology is proposed for the optimal design of each joint. Eventually, an R-joint and an X-joint, optimized for the same prescribed wrench-feasible workspace, are compared on the basis of their maximal actuation forces.
\end{abstract}

Keywords: Revolute joint, Antiparallelogram joint, Tensegrity, Antagonistic forces, Static analysis, Stiffness, Optimal Design

\section{Introduction}

Most robotic manipulators are articulated with revolute joints (R-joints), which are either directly actuated with motors mounted on the joints, or remotely actuated with timing belts or gears. Cables can also be used as a way to transmit motion from the motors mounted on the ground. In cable-driven parallel manipulators (which are not in the scope of this paper), cables are used in place of the legs of a parallel manipulator. In cable-driven serial manipulators, a serial kinematic chain made of rigid links and joints is actuated with a set of cables arranged in parallel [1]. They are interesting solutions when a light-weight design is required and/or physical interactions with the environment are involved. Applications can be found in artificial hands [2], exoskeletons [3] or medical assistance devices. Like in classical robotic manipulators, revolute joints are generally used in cable-driven serial manipulators [1],[4]. In this paper, an antiparallelogram joint, referred to as $\mathrm{X}$-joint, is considered as an alternative choice. The $\mathrm{X}$-joint is less popular than the parallelogram joint and, as far as we know, it has never been used in industrial robots. It was used in knee [5] and bird neck models [6], and in gear trains [7]. The $\mathrm{X}$-shape tensegrity model originally proposed by Snelson [8] has often been used in planar tensegrity mechanisms [6],[9],[10],[11],[12],[13]. In this paper, two antagonistically actuated joints (R-joint and $\mathrm{X}$-joint) are studied. Both joints are equipped with two lateral springs and are actuated with two opposite cables running through the springs. Their static models are derived and 
their static performances are analyzed. A design methodology is proposed, which determines the minimal actuation forces required to reach a prescribed wrenchfeasible workspace (WFW). Finally, an R-joint and an X-joint, optimized for the same WFW, are compared on the basis of actuation forces and stiffness.

\section{Static analysis of the joints}

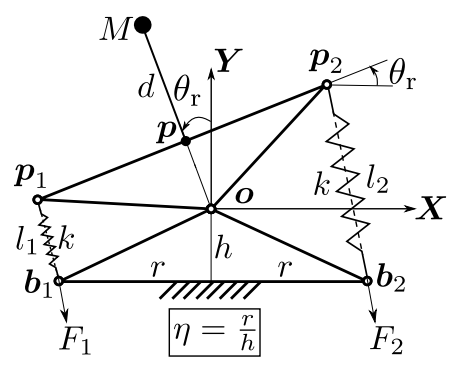

(a) R-joint

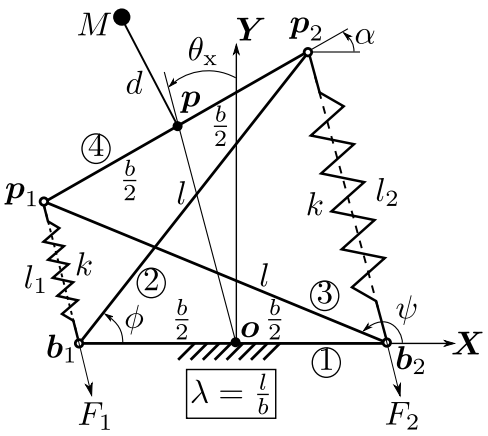

(b) X-joint

Fig. 1: Schematics of the joints under study: R-joint (left) and X-joint (right).

The schematic diagrams of the R-joint and the X-joint under study are shown in Figs. 1a and 1b, respectively. The R-joint is composed of two congruent isosceles triangles, one on top of the other, connected by a revolute joint at $\boldsymbol{o}$. The triangles are defined by two geometric parameters: the semi-base length $r$, and height $h$. The configuration of this joint is described by the orientation angle $\left(\theta_{\mathrm{r}}\right)$ of the upper triangle w.r.t. the vertical as shown in Fig. 1a. On the other hand, the $\mathrm{X}$-joint is composed of three moving links 2, 3, 4 and a fixed link 1, each connected to its neighbours with a revolute joint. The links 1 and 4 are of length $b$, while the other two links are of length $l$. The configuration of the $\mathrm{X}$-joint is defined by the orientation angle $\theta_{\mathrm{x}}$ of the segment linking the midpoints of bars 1 and 4 w.r.t. the vertical as shown in Fig. 1b. Note that the assembly condition requires $l>b$.

Each joint is equipped with a pair of identical springs with spring constant $k$, to impart stiffness into the system. Also, the free-lengths of the springs are assumed to be zero in this study. For both the joints, a point mass $M$ is attached to the segment $\boldsymbol{p}_{1} \boldsymbol{p}_{2}$ at a distance $d$. The linear mass density (i.e., mass per unit length) of the links is represented by $\rho$. Finally, the joints are actuated antagonistically by cables passing through the springs, imparting forces $F_{1}$ and $F_{2}$, respectively, as shown in Fig. 1.

\subsection{Static equilibrium and stiffness of the R-joint}

From Fig. 1a, $l_{1}$ and $l_{2}$ can be expressed in terms of $\theta_{\mathrm{r}}$ as follows:

$$
l_{1}=2\left(h \cos \frac{\theta_{\mathrm{r}}}{2}-r \sin \frac{\theta_{\mathrm{r}}}{2}\right), \quad l_{2}=2\left(h \cos \frac{\theta_{\mathrm{r}}}{2}+r \sin \frac{\theta_{\mathrm{r}}}{2}\right)
$$


The rotation range of the $\mathrm{R}$-joint is limited by singularities due to the actuating cables, in two different ways. Firstly, due to the occurrence of force-closure singularity (see [14]), when the points $\boldsymbol{b}_{i}, \boldsymbol{o}$, and $\boldsymbol{p}_{i}(i=1$ or 2$)$ become collinear. Secondly, due to the vanishing of $l_{1}$ or $l_{2}$, where the direction of force applied by the respective cable becomes ill-defined. It can been shown that the limit of motion is due to force-closure singularity when $r<h$ and due to vanishing of $l_{i}(i=1,2)$ when $r>h$. The rotation range depends on the link lengths and reaches its maximum amplitude ] $-\frac{\pi}{2}, \frac{\pi}{2}$ [ when $r=h$ (see [16] for more details).

Differentiation of the total potential energy of the joint w.r.t. $\theta_{\mathrm{r}}$ yields the equation of static equilibrium, which is of the form: $G_{\mathrm{r}}=\Gamma_{\mathrm{r}}$, where,

$G_{\mathrm{r}}=C \sin \theta_{\mathrm{r}}$, with $C=\frac{1}{3}\left(6 k\left(r^{2}-h^{2}\right)-4 \rho g h\left(r+\sqrt{r^{2}+h^{2}}\right)-3 M g(d+h)\right)$

$\Gamma_{\mathrm{r}}=-F_{1} \frac{\mathrm{d} l_{1}}{\mathrm{~d} \theta_{\mathrm{r}}}-F_{2} \frac{\mathrm{d} l_{2}}{\mathrm{~d} \theta_{\mathrm{r}}}$

The symbol $G_{\mathrm{r}}$ represents the wrench due to gravity and springs, while $\Gamma_{\mathrm{r}}$ represents the external wrench applied by the cables. The forces provided by the cables are limited physically, leading to: $F_{1}, F_{2} \in\left[F_{\min }, F_{\max }\right]$. Since the coefficient of $F_{1}$ (resp. $F_{2}$ ) in $\Gamma_{\mathrm{r}}$ is always negative (resp. positive), the maximal (resp. minimal) boundary of the available wrench $\Gamma_{\max }\left(\operatorname{resp} . \Gamma_{\min }\right)$ is obtained when $F_{1}=F_{\max }$ and $F_{2}=F_{\min }$ (resp. $F_{1}=F_{\min }$ and $\left.F_{2}=F_{\max }\right)$ (see [16]). Considering these limits on $\Gamma_{\mathrm{r}}$, it follows that the equation of static equilibrium can be satisfied only when: $G_{\mathrm{r}} \in\left[\Gamma_{\min }, \Gamma_{\max }\right]$. The range of $\theta_{\mathrm{r}}$ within which this condition is valid is the WFW of the joint.

The joint stiffness $K_{\mathrm{r}}$ is derived upon computing the second derivative of the total potential energy w.r.t. $\theta_{\mathrm{r}}$. We obtain:

$$
K_{\mathrm{r}}=C \cos \theta_{\mathrm{r}}+\frac{1}{2} F_{1}\left(-h \cos \frac{\theta_{\mathrm{r}}}{2}+r \sin \frac{\theta_{\mathrm{r}}}{2}\right)-\frac{1}{2} F_{2}\left(h \cos \frac{\theta_{\mathrm{r}}}{2}+r \sin \frac{\theta_{\mathrm{r}}}{2}\right)
$$

\subsection{Static equilibrium and stiffness of the $\mathrm{X}$-joint}

From Fig. 1b, $l_{1}$ and $l_{2}$ can be obtained in terms of $\theta_{\mathrm{x}}$ as:

$$
l_{1}=-b \sin \theta_{\mathrm{x}}+\sqrt{l^{2}-b^{2} \cos ^{2} \theta_{\mathrm{x}}}, \quad l_{2}=b \sin \theta_{\mathrm{x}}+\sqrt{l^{2}-b^{2} \cos ^{2} \theta_{\mathrm{x}}}
$$

The singularities that limit the motion of the $\mathrm{X}$-joint occur at $\theta_{\mathrm{x}}= \pm \frac{\pi}{2}$, irrespective of the dimensions of the links.

The static equilibrium equation is obtained upon differentiation of the total potential energy of the joint w.r.t. $\theta_{\mathrm{x}}$ as: $G_{\mathrm{x}}=\Gamma_{\mathrm{x}}$, where:

$$
\begin{aligned}
& \left\{\begin{array}{l}
\quad G_{\mathrm{x}}=C_{1} \sin 2 \theta_{\mathrm{x}}+\frac{C_{2} \sin \theta_{\mathrm{x}}\left(2 b^{2} \cos ^{2} \theta_{\mathrm{x}}-l^{2}\right)}{b \sqrt{l^{2}-b^{2} \cos ^{2} \theta_{\mathrm{x}}}} \\
\text { with } C_{1}=2\left(b^{2} k-M g d\right), C_{2}=b g(M+\rho(b+l))
\end{array}\right. \\
& \Gamma_{\mathrm{x}}=-F_{1} \frac{\mathrm{d} l_{1}}{\mathrm{~d} \theta_{\mathrm{x}}}-F_{2} \frac{\mathrm{d} l_{2}}{\mathrm{~d} \theta_{\mathrm{x}}}
\end{aligned}
$$


The symbols $G_{\mathrm{x}}$ and $\Gamma_{\mathrm{x}}$ possess the same physical meaning as $G_{\mathrm{r}}$ and $\Gamma_{\mathrm{r}}$, respectively and the bounds of $\Gamma_{\mathrm{x}}$ are reached for the same force bounds as for the $\mathrm{R}$-joint. The joint stiffness is obtained from the second derivative of the total potential function w.r.t. $\theta_{\mathrm{x}}$. Its expression is not reported here for lack of space, but can be found in [16].

\section{Optimal design of the joints for a specified WFW}

In this study, the link lengths and the spring constant of the joints are considered to be the design variables, while the linear mass density and payload characteristics $(\rho, M, d)$ are treated as parameters whose values are known a priori. The goal is to find optimal designs of the joints, such that the following conditions are met:

- The joint should possess the specified WFW of the general form: $\left[-\theta_{\max }, \theta_{\max }\right]$ with $\theta_{\max }<\frac{\pi}{2}$ to avoid singularities.

- The joint stiffness must be non-negative throughout the WFW for all admissible values of forces satisfying the equation of static equilibrium. Additionally, the stiffness must be equal to a prescribed value $\left(K_{0}>0\right)$ when no actuation forces $\left(F_{1}, F_{2}\right)$ are applied and equal to $\left(K_{1}>0\right)$ at the boundary of the WFW.

- The force required to move the joint must be a minimum.

Due to symmetry of the joints about their respective zero orientations, ensuring $\left[0, \theta_{\max }\right] \in \mathrm{WFW}$, ensures that $\left[-\theta_{\max }, 0\right] \in \mathrm{WFW}$. Similar arguments can be made about the non-negativeness of the stiffness of the joints as well. This makes it sufficient to study just one half of the problem, i.e., $\theta_{\mathrm{r}}>0$ and $\theta_{\mathrm{x}}>0$. In the following, the positive boundary of WFW is denoted by $\theta_{\mathrm{rm}}$ for the R-joint, and $\theta_{\mathrm{xm}}$ for the $\mathrm{X}$-joint. In order to satisfy the conditions listed above, a system of equations and inequalities have been formulated for the two joints as shown in Table 1 (assuming $F_{\min }=0$ ). Physically, the first two conditions ensure that no singularities occur within the WFW and that the positive boundary of the WFW is formed by the intersection of the curves $G_{\mathrm{r}}$ (resp. $G_{\mathrm{x}}$ ) and $\Gamma_{\max }$. The third and fifth conditions ensure that the stiffness of the joint is equal to $K_{0}$ in the absence of applied forces, and equal to $K_{1}$ at the boundary of the WFW. The remaining condition ensures that the joint possesses a non-negative stiffness at the zero orientation when maximum forces are applied. The ratio of link lengths: $\eta\left(=\frac{r}{h}\right)$ for the R-joint and $\lambda\left(=\frac{l}{b}\right)$ for the $\mathrm{X}$-joint have been introduced into the formulation, eliminating the variables $h$ and $l$, respectively. This is because the ratio provides more insights into the problem and also simplifies the resulting expressions considerably. The conditions in Table 1 are then derived in terms of the joint parameters. Their expressions are not reported here for lack of space, but can be found in [16]. Using these expressions, design optimization problems for the R-joint and the $\mathrm{X}$-joint are formulated and solved in the following. 
Table 1: Formulation of the stipulated conditions for the R-joint and the X-joint.

\begin{tabular}{|lr|lc|}
\hline \multicolumn{1}{|c|}{ R-joint } & \multicolumn{1}{|c|}{ X-joint } \\
\hline$l_{1}\left(\theta_{\mathrm{rm}}\right)>0$ & $(8 \mathrm{a})$ & No singularities when $\left(\theta_{\mathrm{xm}}<\frac{\pi}{2}\right)$ & $(9 \mathrm{a})$ \\
$G_{\mathrm{r}}\left(\theta_{\mathrm{rm}}\right)+F_{\max } \frac{\mathrm{d} l_{1}}{\mathrm{~d} \theta_{\mathrm{r}}}\left(\theta_{\mathrm{rm}}\right)=0$ & $(8 \mathrm{~b})$ & $G_{\mathrm{x}}\left(\theta_{\mathrm{xm}}\right)+F_{\max } \frac{\mathrm{d} l_{1}}{\mathrm{~d} \theta_{\mathrm{x}}}\left(\theta_{\mathrm{xm}}\right)=0$ & $(9 \mathrm{~b})$ \\
$K_{\mathrm{r}}\left(\theta_{\mathrm{r}}=0, F_{1}=0, F_{2}=0\right)=K_{0}$ & $(8 \mathrm{c})$ & $K_{\mathrm{x}}\left(\theta_{\mathrm{x}}=0, F_{1}=0, F_{2}=0\right)=K_{0}$ & $(9 \mathrm{c})$ \\
$K_{\mathrm{r}}\left(\theta_{\mathrm{r}}=0, F_{1}=F_{\max }, F_{2}=F_{\max }\right) \geq 0$ & $K_{\mathrm{x}}\left(\theta_{\mathrm{x}}=0, F_{1}=F_{\max }, F_{2}=F_{\max }\right) \geq 0$ \\
& $(8 \mathrm{~d})$ & $(9 \mathrm{~d})$ \\
$K_{\mathrm{r}}\left(\theta_{\mathrm{r}}=\theta_{\mathrm{rm}}, F_{1}=F_{\max }, F_{2}=0\right)=K_{1}$ & $K_{\mathrm{x}}\left(\theta_{\mathrm{x}}=\theta_{\mathrm{xm}}, F_{1}=F_{\max }, F_{2}=0\right)=K_{1}$ \\
& $(8 \mathrm{e})$ & $(9 \mathrm{e})$ \\
\hline
\end{tabular}

\subsection{Optimal design of the R-joint}

It is noted that the set of design variables of the R-joint is formed by $r, \eta$, and $k$. Using Eq. (4) in Eq. (8c) results in the condition: $C=K_{0}$. From the expression of $C$ provided in Eq. (2), it is possible to solve for $k$ as:

$$
k=\frac{\eta^{2}}{2 r^{2}\left(\eta^{2}-1\right)}\left(K_{0}+\frac{4 r^{2}}{3 \eta^{2}} \rho g\left(\eta+\sqrt{\eta^{2}+1}\right)+M g\left(d+\frac{r}{\eta}\right)\right)
$$

From the above equation, it is clear the condition $\eta>1$ is necessary to ensure that $k$ remains positive. Also, from the inequality in Eq. (8a), the condition: $\eta<\cot \frac{\theta_{\mathrm{rm}}}{2}$ is obtained. By substituting $C=K_{0}$ in Eq. (8b), the force required to reach the specified WFW is obtained as: $F_{\max }=\frac{K_{0} \eta \sin \theta_{\mathrm{rm}}}{r\left(\sin \frac{\theta_{\mathrm{rm}}}{2}+\eta \cos \frac{\theta_{\mathrm{rm}}}{2}\right)}$. Using the above conditions, it can be shown that the inequality in Eq. (8d) is satisfied by default, when $\eta>1$, for all values of $\left.\theta_{\mathrm{rm}} \in\right] 0, \frac{\pi}{2}$ [ (see [16] for details). Further, substituting $C=K_{0}$ and the above expression of $F_{\max }$ in Eq. (8e), leads to:

$$
\eta=\frac{K_{0}\left(1-\cos \theta_{\mathrm{rm}}\right)+2 K_{1}}{K_{0}\left(1+\cos \theta_{\mathrm{rm}}\right)-2 K_{1}} \tan \left(\frac{\theta_{\mathrm{rm}}}{2}\right)
$$

The above equation provides a simple relationship between the design specifications $\left(\theta_{\mathrm{rm}}, K_{0}, K_{1}\right)$ and $\eta$. This is quite interesting because for a given set of specifications, the ratio of link dimensions remains fixed, irrespective of the payload $(M, d)$ and the material of the links $(\rho)$. It is noted that the specifications $K_{0}, K_{1}$, and $\theta_{\text {rm }}$, must allow the bounds on: $\left.\eta \in\right] 1$, $\cot \frac{\theta_{\mathrm{rm}}}{2}$ [ to be satisfied, for the existence of feasible designs. Substituting for $\eta$ from Eq. (11) into the expression of $F_{\max }$ and $k$ (Eq. (10)), both of them can be obtained in terms of $r$, which is the only remaining variable. Thus, to minimize the force required to move the joint, the following optimization problem is posed:

$$
\begin{array}{ll}
\underset{r}{\text { Minimize }} & F_{\max }(r)=\frac{\left(K_{0}\left(1-\cos \theta_{\mathrm{rm}}\right)+2 K_{1}\right)}{r} \sin \left(\frac{\theta_{\mathrm{rm}}}{2}\right) \\
\text { subject to } & r \in[0.025,0.1], \\
& k \in[0,2000],
\end{array}
$$


where $r$ is the design variable of this problem. The constraint on $\eta$ is not mentioned in the problem as it should be satisfied by the choice of $K_{0}, K_{1}$, and $\theta_{\mathrm{rm}}$. Bounds on the variables $r$ and $k$ have been imposed in the problem due to practical considerations, such as, availability of corresponding components in the market and ease of fabrication/assembly. Using Eq. (10), equivalent algebraic conditions on $r$, corresponding to bounds on $k$ can be obtained. These conditions would be used for defining the feasible design space of the R-joint. The first-order necessary condition for $F_{\max }$ to achieve a local minima requires the vanishing of its derivative w.r.t. $r$. However, it is found that $\frac{\mathrm{d} F_{\max }}{\mathrm{d} r}=$

$-\frac{\left(K_{0}\left(1-\cos \theta_{\mathrm{rm}}\right)+2 K_{1}\right)}{r^{2}} \sin \left(\frac{\theta_{\mathrm{rm}}}{2}\right)$, is negative for all feasible values of the design variables and parameters. This implies that $F_{\max }$ decreases with increase in $r$, and its minimum value would occur when $r$ is as large as possible, while satisfying the constraints specified in Eq. (12). Further information on the minimum value of force and the corresponding design variables can be obtained by studying the behavior of $F_{\max }$ inside the design space, as illustrated in Fig. 2a.

\subsection{Optimal design of the $\mathrm{X}$-joint}

It is noted that the set of design variables of the $\mathrm{X}$-joint is formed by: $b, \lambda$, and $k$. Using Eq. (9b) and Eq. (9e), it is possible to obtain the expression of $F_{\max }$ reported in Eq. (15), and a relation between $C_{1}$ and $C_{2}$ of the form: $C_{1}=$ $\gamma_{1} K_{1}+\gamma_{2} C_{2}$, where $\gamma_{1}$ and $\gamma_{2}$ are functions of $\lambda$ and $\theta_{\mathrm{xm}}$, which are suppressed here due to lack of space (see [16] for details). Substituting this relation in Eq. (9c) leads to:

$$
2 \gamma_{1} K_{1}+\gamma_{3} C_{2}-K_{0}=0, \text { where } \gamma_{3}=2 \gamma_{2}-\frac{\lambda^{2}-2}{\sqrt{\lambda^{2}-1}}
$$

Substituting the expression of $C_{2}$ from Eq. (6) into Eq. (13), one obtains a quadratic equation in $b$ as: $\rho g \gamma_{3}(\lambda+1) b^{2}+M g \gamma_{3} b+2 \gamma_{1} K_{1}-K_{0}=0$. Considering $b>0$, this equation provides a unique solution to $b$ in terms of $\lambda$ as follows:

$$
b=\frac{\sqrt{\gamma_{3}^{2} g^{2} M^{2}-4 \gamma_{3} g(\lambda+1) \rho\left(2 \gamma_{1} K_{1}-K_{0}\right)}-\gamma_{3} g M}{2 \gamma_{3} g(\lambda+1) \rho}
$$

Using this expression of $b$, it is possible to obtain $C_{2}$ (from Eq. (6)), and then $C_{1}$ in terms of $\lambda$. Further, from the definition of $C_{1}$ (see Eq. (6)), the spring constant $k$ can be found as: $k=\frac{C_{1}+2 M g d}{2 b^{2}}$. Using Eq. (13), it can be shown that the inequality in Eq. (9d) reduces to: $K_{0}+\frac{2 b F_{\max }}{\sqrt{\lambda^{2}-1}} \geq 0$, which is satisfied by default, since all of its terms are positive. Thus, the optimization problem for 
the design of the $\mathrm{X}$-joint is posed as follows:

$$
\begin{array}{ll}
\underset{\lambda}{\operatorname{Minimize}} & F_{\max }(\lambda)=\frac{C_{2} \lambda^{4} \sin \theta_{\mathrm{xm}} \tan ^{2} \theta_{\mathrm{xm}}+K_{1} \tan \theta_{\mathrm{xm}}\left(\lambda^{2}-\cos ^{2} \theta_{\mathrm{xm}}\right)^{3 / 2}}{b \cos \theta_{\mathrm{xm}}\left(\left(\lambda^{2}-\cos ^{2} \theta_{\mathrm{xm}}\right)^{3 / 2}-\sin ^{3} \theta_{\mathrm{xm}}\right)} \\
\text { subject to } & k \in[0,2000], \\
& b \in[0.05,0.2], \\
& \lambda \in] 1,5],
\end{array}
$$

where $\lambda$ is the only design variable in this problem $\left(\lambda=\frac{l}{b}\right)$. The bounds on $k$ and $b$ must be transferred to $\lambda$, to define the feasible design space for $\mathrm{X}$-joint. However, due to the complicated functional relationship between variables, an equivalent set of algebraic conditions on $\lambda$ could not be derived. Nevertheless, from a plot of $b$ (resp. $k$ ) against $\lambda$, it is possible to identify the feasible regions visually, and then compute the corresponding limiting points numerically, to define the feasible design space (see Fig. 2b).

As in the previous case, the first-order necessary condition for $F_{\max }$ to attain a minima is obtained from the condition: $\frac{\mathrm{d} F_{\max }}{\mathrm{d} \lambda}=0$. The corresponding algebraic expression is too huge to be reported in this paper (see [16] for details). Solution to the said equation would provide the stationary points of $F_{\max }$. Firstly, it is essential to check if there are solution(s) that satisfy all the constraints specified in Eq. (15). Secondly, such solutions must be classified as a minimum or a maximum or an inflection point, through the second derivative test or by inspecting the plot of $F_{\max }$ against $\lambda$, as illustrated in Fig. 2b. In case several minima exist within the feasible design space, then the one that corresponds to the least value of $F_{\max }$ must be chosen. On the other hand, if no minima exists, then the solution to this problem must be at/near a boundary of the feasible design space, depending on whether the boundary point is included or not.

\section{Numerical examples and inferences}

All the links are considered to be 3D printed using ABS material with a circular cross section of diameter equal to $0.01 \mathrm{~m}$, as in [15]. Consequently, the linear mass density $(\rho)$ of the links is found to be $0.0825 \mathrm{Kg} / \mathrm{m}$. Point mass $(M)$ and offset $(d)$ values are considered to be: $M=0.2 \mathrm{Kg}$ and $d=0.25 \mathrm{~m}$, respectively. Ideally, a suitable value for the prescribed stiffness $\left(K_{0}, K_{1}\right)$ should be determined through experiments to estimate the amount of disturbance the joint must withstand. In this study, these values are chosen to be: $K_{0}=1 \mathrm{Nm} / \mathrm{rad}$ and $K_{1}=0.6 \mathrm{Nm} / \mathrm{rad}$, for both the joints after verifying that the necessary conditions discussed in Sections 3.1 and 3.2 are satisfied. The WFW is stipulated to be $\left[-\frac{5 \pi}{18}, \frac{5 \pi}{18}\right]$ (i.e., $\left.\left[-50^{\circ}, 50^{\circ}\right]\right)$ for the two joints.

Using the above data, $F_{\max }$ is plotted in the design space $(r)$ of the R-joint as shown in Fig. 2a. It is observed that the feasible design space is formed by the constraint: $0.025 \leq r \leq 0.1$ (red). From the plot, $F_{\max }$ attains its minimum 


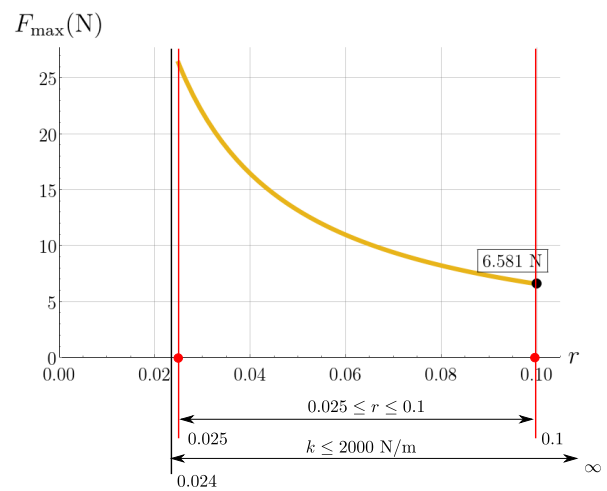

(a) R-joint: Plot of $F_{\max }$ with $r$ and boundary of constraints $0.025 \leq r \leq 0.1$ (red) and $k \leq 2000$ (black)

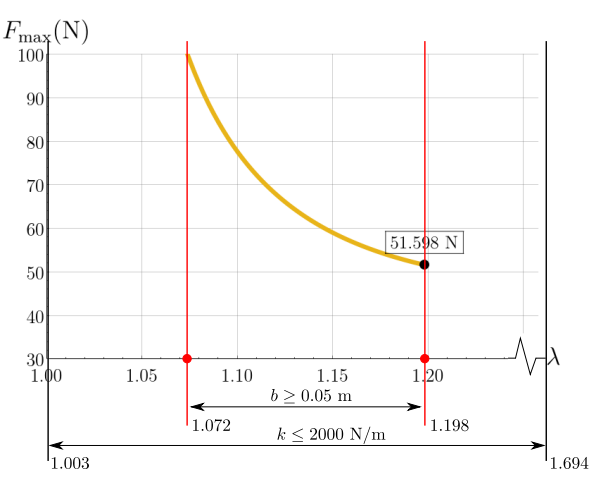

(b) X-joint: Plot of $F_{\max }$ with $\lambda$ and boundary of constraints $b \geq 0.05$ (red) and $k \leq 2000$ (black)

Fig. 2: Variation of $F_{\max }$ inside the feasible design space of the joints.

value of $6.581 \mathrm{~N}$ when: $r=0.1 \mathrm{~m}, \eta=1.6399(h=r / \eta=0.061)$, and $k=$ $129.2487 \mathrm{~N} / \mathrm{m}$.

In Fig. $2 \mathrm{~b}, F_{\max }$ is plotted against the design variable $(\lambda)$ of the $\mathrm{X}$-joint. It is observed that the feasible design space is reduced to $\lambda \in[1.072,1.198]$, by the constraint $b \geq 0.05$ (red). It is found that the equation $\frac{\mathrm{d} F_{\max }}{\mathrm{d} \lambda}=0$, results in $\lambda=1.3$, which is outside the feasible domain. Thus, from the plot, the minimum value of $F_{\max }=51.598 \mathrm{~N}$ occurs when $\lambda=1.1982, b=0.05 \mathrm{~m}$ $(l=\lambda b=0.0599)$, and $k=287.2395 \mathrm{~N} / \mathrm{m}$.

The optimal designs, corresponding forces, and bounds on stiffness of the joints are presented in Fig. 3. From the obtained results, the following inferences are made:

- From the link dimensions, it is observed that the width (resp. height) of the $\mathrm{R}$-joint is 4 (resp. about 3.7) times more than that of the $\mathrm{X}$-joint. The mass of the R-joint (without payload) is found to be $0.085 \mathrm{Kg}$, which is about 4.5 times the mass of the X-joint, computed to be $0.018 \mathrm{Kg}$.

- The stiffness upper bound of the R-joint (resp. X-joint) is reached when one of the applied forces is equal to $F_{\min }\left(\right.$ resp. $\left.F_{\max }\right)$. This shows that increasing the applied forces decreases the stiffness of the R-joint, while it increases the stiffness of the X-joint.

- The force required to move the $\mathrm{R}$-joint is lower (about $1 / 8$ times) when compared to the $\mathrm{X}$-joint. This implies that the $\mathrm{R}$-joint is more sensitive to small changes in the applied forces.

- The stiffness value of the $\mathrm{X}$-joint is higher (about 9 times) when compared to the R-joint, around the zero orientation. Additionally, the distribution of stiffness is more uniform throughout the WFW for the R-joint, while relatively large differences in stiffness is observed for the $\mathrm{X}$-joint inside its WFW, 


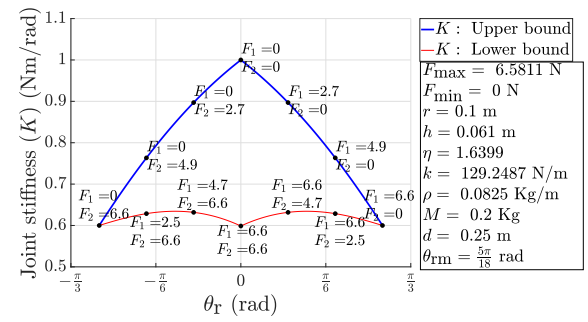

(a) R-joint (Forces in N)

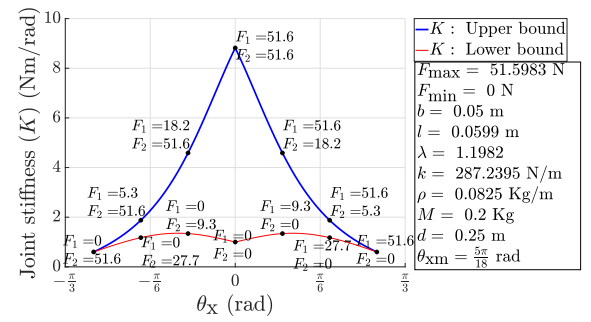

(b) X-joint (Forces in $\mathrm{N}$ )

Fig. 3: Plots of stiffness bounds corresponding to the optimal design of the joints for a specified WFW of $\left[-\frac{5 \pi}{18}, \frac{5 \pi}{18}\right]$ with a payload: $M=0.2 \mathrm{Kg}, d=0.25 \mathrm{~m}$.

between the zero orientation and the boundary. Moreover, the difference between the minimal and maximal stiffness is greater for the $\mathrm{X}$-joint, especially around the zero orientation. The $\mathrm{X}$-joint is thus more suitable for variable stiffness.

- The value of spring constant $(k)$ required is about two times greater for the $\mathrm{X}$-joint than for the R-joint.

\section{Conclusion}

The static analysis of two antagonistically actuated joints with a point mass payload has been conducted in this study: the revolute $(\mathrm{R})$ joint and the antiparallelogram (X) joint. An optimal design strategy has been proposed to minimize the actuation forces for a prescribed wrench-feasible workspace (WFW) with a prescribed stiffness at rest and at the WFW bounds. The joints have been compared in terms of their actuation forces and stiffness, when they are designed to possess the same WFW. It is found that the R-joint is heavier, requires lower forces, is more sensitive to applied forces, and possesses a more uniform stiffness distribution throughout its workspace. On the other hand, the X-joint reaches a higher stiffness near its zero orientation and exhibits relatively large variations in stiffness within its WFW. The stiffness of the X-joint can be easily increased by increasing the forces magnitude. For the R-joint, in contrast, the stiffness decreases when forces magnitude increases. In the future, the comparative study and design strategy will take into account the dynamics and will be extended to manipulators with several $\mathrm{R}$ - and $\mathrm{X}$-joints in series.

Acknowledgement This work was conducted with the support of the French National Research Agency (AVINECK Project ANR-16-CE33-0025).

\section{References}

1. Ramadoss, V., Zlatanov, D., Zoppi, M.: Kinematic and Workspace Analysis of Minimally Routed Cable Driven Open Chains. In: Proc. 15th World Congress in Mechanism and Machine Science, IFToMM'2019, Krakow, Poland (2019) 
2. Baril, M., Laliberté, T., Guay, F., Gosselin, C.: Static Analysis of SingleInput/Multiple-Output Tendon-Driven Underactuated Mechanisms for Robotic Hands. In: Proc. ASME 2010 IDETC Conference, vol. 2: 34th Annual Mech. and Rob. Conference, Parts A and B, pp. 155-164, Montreal, Quebec, Canada (2010)

3. Mao, Y., Agrawal, S. K.: Design of a Cable-Driven Arm Exoskeleton (CAREX) for Neural Rehabilitation. IEEE Transactions on Robotics 28(4), 922-931 (2012)

4. Chen, B., Cui, Z., Jiang, H.: Producing negative active stiffness in redundantly actuated planar rotational parallel mechanisms. Mechanism and Machine Theory 128, 336-348 (2018)

5. Hamon, A., Aoustin, Y., Caro, S.: Two walking gaits for a planar bipedal robot equipped with a four-bar mechanism for the knee joint. Multibody System Dynamics 31(3), 283-307 (2014)

6. Furet, M., Wenger, P.: Kinetostatic analysis and actuation strategy of a planar tensegrity 2-X manipulator. ASME Journal of Mechanisms and Robotics 11(6) (2019) doi:10.1115/1.4044209.

7. Zhang, X., Deng, H., Wang, X., Pan, C., Xu, H.: Use of Anti-parallelogram Linkage Mechanism and Ordinary Gear Train for Power Transmission on a Rotary Engine. In: Proc. Third Int. Conf. on Mechanic Aut. and Control Eng., pp. 1041-1044 (2012)

8. Snelson, K.: Continuous Tension, Discontinuous Compression Structures. US Patent No. 3,169,611 (1965)

9. Arsenault, M., Gosselin, C.M.: Kinematic, static and dynamic analysis of a planar 2dof tensegrity mechanism. Mechanism and Machine Theory 41(9), 1072-1089 (2006)

10. Wenger, P., Chablat, D.: Kinetostatic analysis and solution classification of a class of planar tensegrity mechanisms. Robotica 37(7), 1214-1224 (2019)

11. Boehler, Q., Charpentier, I., Vedrines, M.S., Renaud, P.: Definition and computation of tensegrity mechanism workspace. ASME Journal of Mechanisms and Robotics 7(4) (2015)

12. Chen, S., Arsenault, M.: Analytical Computation of the Actuator and Cartesian Workspace Boundaries for a Planar 2-Degree-of-Freedom Translational Tensegrity Mechanism. ASME Journal of Mechanisms and Robotics 4(1) (2012)

13. Bakker, D.L., Matsuura, D., Takeda, Y., Herder, J.L.: Design of an environmentally interactive continuum manipulator. In: Proc. 14th World Congress in Mechanism and Machine Science, IFToMM'2015, Taipei, Taiwan (2015)

14. Diao, X., Ma, O., Lu, Q.: Singularity Analysis of Planar Cable-Driven Parallel Robots. In: 2008 IEEE Int. Conf. on Rob., Aut. and Mechatronics, pp. 272-277 (2008)

15. Furet, M., van Riesen, A., Chevallereau, C., Wenger, P.: Optimal design of tensegrity mechanisms used in a bird neck model. In: EuCoMeS 2018, B. Corves, P. Wenger, and M. Hüsing, Eds., vol. 59, pp. 365-375. Cham: Springer Int. Publishing (2019)

16. Muralidharan, V.: Static analysis and design of revolute joint and antiparallelogram joint. Technical report, LS2N-CNRS, Ecole Centrale de Nantes (2020) http://dx.doi.org/10.13140/RG.2.2.17902.77120/1 